\title{
LOW FREQUENCY ELECTROMAGNETIC FIELDS: FRIEND OR FOE?
}

The issue of electromagnetic fields and their effects on human health has long been discussed within the scientific community. The lack of undisputable evidence confirming the dangers of these fields leads to great uncertainty and public concerns. On the other hand, positive effects and possible therapeutic use of the mentioned fields has also been explored and currently hints at feasible application thereof in regenerative and therapeutic applications. The following article provides a balanced insight into the issue at hand and provides details of recently proposed mechanisms of actions.

Keywords: Electromagnetic field, Bio-electromagnetism, Electric properties of Cells, Electromagnetic Smog, Electromagnetic Therapy.

\section{Introduction}

Electricity in every conceivable form has become an integral part of our everyday lives. Many daily tasks that we perform depend on it and there is no sign that the current progress will slow down any time soon. However, because living organisms evolved over billions of years in a world with a reasonably weak magnetic field and with few electromagnetic (EM) energy sources, they interacted with and adapted to this specific range of EM fields (EMF). Thus, due to the widespread presence of new EMF sources, health issues have been brought up because we still lack a thorough understanding of the fundamental properties of these fields.

Since the first observations of health disturbances related to long-term exposure to electric power line configurations were reported 20 years ago, there has been a goodly amount of scientific discussion about the bioeffects of extremely low frequency magnetic fields (ELF-MF). While the basics of electromagnetic interaction with materials were elucidated over a century ago and stated as the well-known Maxwell's equations, the application of these basics to biological systems is very difficult because of the extreme complexity and multi-level organization in living organisms. This difficulty has slowed the progress of understanding the EM bioeffects and phenomena that might directly influence our health. Nevertheless, it is clear that any explanation is beyond the scope of conventional physics and that we must concentrate our efforts at the cellular and subcellular level, where the smallest of changes could trigger the greatest of damage, Jakusova [1].

The purpose of this article is to attempt to give a balanced overview of the issue at hand, including review of select health studies in this field, proposal of therapeutic applications and elucidation of novel mechanisms of action based on the modulation of membrane voltage, paving the road to forthcoming venues for guiding morphogenesis both in tissue engineering and in regenerative medicine.

\section{Health issues and considerations}

Health concerns are especially associated with carcinogenic or co-carcinogenic effects of electromagnetic fields. Herein reviewed studies indicate no clear link between low frequency EMF (LFEMF) and increased cancer risk, although childhood leukemia increase has been documented in several epidemiological and toxicological studies.

Karipidis et al. [2] examined low and high grade glioma risk in various patients. A group of 414 histologically confirmed glioma cases and a group of 421 controls from Melbourne, Australia were matched by age, sex and postcode of residence. Results indicate increased glioma and HGG (high-grade glioma) cases in expert hygienist methodologies, though not of statistical significance.

Incidence of kinase 4 inhibitor gene ( $\left.\mathrm{p} 18^{\mathrm{INK} 4 \mathrm{C}}\right)$ deletion and/or mutation in electric energy workers was explored by Erdal et al. [3]. A choice of 31 male electric workers and 30 healthy males between $30-40$ years of age and in the same geographical area and with similar lifestyles was selected. While exon 1 band migration was indifferent in all participants, exon 2 of two electric workers was slow in migration with respect to the controls. However, the limited results do not suggest any significant risk of cancer increase.

Both Johansen and Olsen [4] and [5] conducted a study among male and female electric utility workers in Denmark. The total number of subjects was 32 006, and while increased cancer

\footnotetext{
* Ivo Cap, Jan Barabas

Faculty of Electrical Engineering, University of Zilina, Slovakia, E-mail: ivo.cap@fel.uniza.sk
} 
was predicted, only slightly higher rates of occurrence were reported and no excess of brain cancer, leukemia or breast cancer were reported.

Cumulative effects of both electric and magnetic fields were investigated by Miller et al. [6]. In their report the authors observed an increase in leukemia, especially due to mutual electric and magnetic field exposure. However, in another study involving 764 adults, Willett et al. [7] found little evidence between occupational EM exposure and acute leukemia.

Childhood leukemia accounts for approximately one third of all child cancers. A summary of select childhood leukemia studies is presented in Table 1:

Childhood leukemia studies

Table 1

\begin{tabular}{|c|c|l|}
\hline Study conductor & Number of subjects & \multicolumn{1}{|c|}{ Results } \\
\hline Verkasalo et al.[8] & $\begin{array}{c}35 \text { cases, } \\
<17 \text { years old }\end{array}$ & $\begin{array}{l}\text { General increase of leukemia } \\
\text { risk; brain tumor (only boys) }\end{array}$ \\
\hline McBride et al.[9] & $\begin{array}{c}596 \text { cases, } \\
<15 \text { years old }\end{array}$ & Elevated risk in all subjects \\
\hline Savitz et al.[10] & $\begin{array}{c}448 \text { cases, } \\
<15 \text { years old }\end{array}$ & Elevated risk in all subjects \\
\hline Linen et al.[11] & $\begin{array}{c}1026 \text { cases, } \\
<19 \text { years old }\end{array}$ & No correlated risk increase \\
\hline Schuz et al.[12] & $\begin{array}{c}489 \text { cases, } \\
\text { N/A years old }\end{array}$ & $\begin{array}{l}\text { Moderate increase in } \\
\text { childhood leukemia, though } \\
\text { not statistically significant }\end{array}$ \\
\hline
\end{tabular}

The exact mechanism of action is unknown, although a melatonin-based hypothesis was proposed by Henshaw and Reiter [13], in which magnetic fields from power lines suppress the nocturnal melatonin production of the pineal gland. Moreover, mutual geneenvironment interactions need also be considered.

Cellular and animal studies, on the other hand, provide no evidence of direct DNA damage or tumor growth. Another reason to consider is the abstractness when comparing in-vitro and in-vivo setups, whereby the former lack natural reparatory and regulatory mechanisms present in their natural environments. Other physiological effects, such as heart rate variability, melatonin suppression, sleep disturbances or anomalous EEG activity, while reported, do lack any consistent results and raise questions whether these are solely tied to electromagnetic exposure or rather represent a risk cofactor.

\section{Emerging regenerative and therapeutic methods}

Over the past years, numerous studies have observed positive effects of controlled low frequency electromagnetic field exposure. The following studies highlight two most prominent regenerative applications - soft tissue and bone repair although they have not explained or quantified the observed effects in a satisfactory fashion. However they incited further research in this area and possible mechanisms of actions are discussed in detail further down this article.

The viability of ELF EMFs in soft tissue regeneration after injury was investigated by Vianale et al. [14]. In their study they applied ELF magnetic fields ( $1 \mathrm{mT}$ intensity, $50 \mathrm{~Hz}$ frequency) to human keratinocyte cell line $(\mathrm{HaCaT})$ and evaluated keratinocyte proliferation and production of various proinflammatory chemokines. Accelerated growth was observed after 48 hours of exposure when compared to the control group, but no difference in cell viability was detected. Proinflammatory chemokine production was reduced significantly after 72 hours of exposure. Especially sensitive to the applied field was the NF-kB signaling pathway which decreased to almost non-detectable levels after just one hour of exposure, inversely correlated with cell density. These findings indicate the possible favorable role of ELF electromagnetic fields in wound healing through keratinocyte growth stimulation and reduction of chemokines.

Effectiveness of ELF electromagnetic fields in modifying specific biochemical properties of human skin keratynocytes $(\mathrm{HaCaT})$ was also evaluated by Lisi et al. [15]. In their ventures, the authors applied $7 \mathrm{~Hz}$ ELF electromagnetic fields with intensities of 100 $\mu \mathrm{T}$ for one hour, twice daily, which resulted in both shape and morphological changes, which are attributed to different actin distribution. The authors, based on evidence of increased expression of both differentiation and adhesion markers (involucrin, $\beta$-catenin) further conclude that higher level keratinocyte expression was achieved.

Lisi et al. [15] reported similar observations as those by Cricenti et al. [16]. Infrared wavelength-selective scanning near-field optical microscopy was again used to examine $\mathrm{HaCaT}$ cells post 24 hour exposure to alternating magnetic fields (sinusoidal shape, $1 \mathrm{mT}$ intensity, $50 \mathrm{~Hz}$ frequency). Reflectivity studies at both wavelengths $\left(\lambda_{1}=6.45 \mu \mathrm{m}\right.$ and $\left.\lambda_{2}=3.04 \mu \mathrm{m}\right)$ univocally reveal an increase of newly synthesized protein and different phospholipids distribution. This finding coincides with a previous study conducted by the same authors and confirms increased segregation in the cell membrane of the sulfur rich protein marker B4 integrin. Additional structural and morphological changes were also detected which lead to increase in adhesion area between individual cells.

Potential therapeutic application of pulsed electromagnetic fields in rheumatoid arthritis (RA) was evaluated by Selvam et al. [17]. RA was induced in rats using heat-killed Mycobacterium tuberculosis. Observed were increased levels of lipid peroxides and depletion of antioxidant enzymes, however more interesting is the decrease of plasma membrane $\mathrm{Ca}^{2+}$ ATPase (PMCA) activity and elevated levels of intracellular $\mathrm{Ca}^{2+}$ and prostaglandin E2 levels. The affected limb was then exposed to $5 \mathrm{~Hz}, 4 \mu \mathrm{T}$ PEMF for 90 minutes, which resulted in restoration of the altered parameters and a pronounced antiexudative effect. The reasoning behind this is through the stabilizing effect of pulsed electromagnetic fields on cellular membranes as experienced via PMCA and $\mathrm{Ca}^{2+}$ level 
restoration leading to inhibition of prostaglandin $E_{2}$ levels, indicating a possible use in supportive treatment of human rheumatoid arthritis.

Adenosine receptor affinity and density in view of PEMFs was explored by Varani et al. [18]. The authors discovered that the $75 \mathrm{~Hz}, 1 \mathrm{mT}$ generated field mediated an upregulation of certain adenosine receptors and leads to a functional increase thereof in bovine chondrocytes and fibroblast-like synoviocytes, without altering adenosine affinity and drug-receptor interactions. These finding suggest that PEMFs could positively influence inflammatory joint diseases.

Possible use of various ELF-EMF types in bone fracture therapy was explored by Zhang et al. [19]. Four electromagnetic field types were investigated: rectangular (REMF), triangular (TEMF), sinusoidal (SEMF) and pulsed (PEMF), all of which showed different effects (proliferation, differentiation, mineralization) on osteoblast cells obtained from rat calvaria bones.

Wei et al. [20] applied PEMFs with a frequency of $48 \mathrm{~Hz}$ and intensity of $1.55 \mathrm{mT}$ to both rat-derived osteoblast cells and MC3T3E1 cells. Based on their experiments, the authors noted that the used PEMFs "did not affect proliferation and differentiation of the osteoblast-like MC3T3-E1 cells, however promoted proliferation, decreased differentiation at proliferation stage and promoted differentiation at differentiation stage of primary osteoblast cells".

\section{A behind the scenes look}

There are numerous cell types in living organisms, some terminally differentiated (e.g. neurons), while others maintain their proliferative status throughout the whole life cycle of the organism. In case of wound or bone fracture healing, initial rapid proliferation is necessary in order to restore the functional integrity of the organism. During this period, however, the disruption of the cell cycle at specific checkpoints may lead to uncontrolled division of cells. On the other hand, a unique opportunity also arises that allows us to bioelectrically (in addition to already present endogenous bioelectric signals) control the mitotic activity via targeted transmembrane voltage variations. This approach presents a plausible explanation of findings in the previously mentioned studies and a tractable technique for functional experiments.

Membrane potential $\left(V_{m e m}\right)$ changes vary with cell type, ranging from millisecond timeframes seen in neurons and muscle cells, to possibly minutes and even days in diurnal cycles, in which case we speak of steady-state membrane potentials. The actual transmembrane voltage is the result of numerous channels and pumps, which segregate ions across the cell surface and are governed by the concentration gradient and charge distribution. To be able to control the cellular Vmem one must first fully understand the endogenous mechanisms responsible for modulating specific ion channels and transporters and subsequently appropriately, and in a controlled fashion, alter the course of such actions in a favorable way.

Numerous studies suggest that membrane potential is a key regulator of proliferation in a number of cell types. Furthermore, specific modulation of $V_{\text {mem }}$ is required for both $\mathrm{G} 1 / \mathrm{S}^{1)}$ phase and $\mathrm{G} 2 / \mathrm{M}^{2)}$ phase transitions. In this context, inhibition of $\mathrm{G} 1 / \mathrm{S}$ progression can be achieved in specific cell types, such as lymphocytes, astrocytes, fibroblasts and Schwann cells via membrane depolarization. The ensuing $\mathrm{S}$ phase initiation relies on hyper-polarization and omission thereof results in a so-called reversible cell cycle arrest, which has been documented in B cell lymphocytes, amongst others. Expression of various proteins was also effected, including IL-1/2 and transferring, both of which are implicated in cell cycle control.

On the other hand, the G2/M transition relies on the depolarization of the plasma membrane. In view of our current understanding, the proposed models outline a rhythmic oscillation of membrane potential throughout the cell cycle, with a spike in hyperpolarization occurring before DNA synthesis followed by a prolonged period of depolarization necessary for mitosis. The exact threshold of $V_{\text {mem }}$ necessary to drive cells though proliferative stages is not known, and is likely to vary between cell type and differentiation stage [21].

This lack of practical knowledge could be one of the reasons behind the many inconsistencies encountered in currently available studies, and places emphasis on future research in this area. However, one should note that our current understanding of this issue is still rudimentary and far from complete.

The transmembrane voltage is indeed only one variable in the whole process of cell division. Additionally, temporal variations must be added to existing models of signaling systems due to $V_{\text {mem }}$ oscillations occurring during the cell cycle. Also of notable importance is the assumption, or rather oversimplification that $V_{\text {mem }}$ is indicative of the whole cell - whereas in reality, distinct domains of membrane voltage around the cell periphery exist in forms of micro-domains, presumably established by distinct population of channel/pump proteins. Finally, while the transmembrane voltage is the best-known and most tractable of the cellular bioelectric parameters, sub-cellular organelles such as mitochondria or nuclei all possess a transmembrane voltage of their own. Future efforts must concentrate on subcellularly-targeted voltage reporter proteins, functionally altering the bioelectric signaling in distinct intracellular locales [21].

\footnotetext{
1) The G1/S transition is a stage in the cell cycle at the boundary between the G1 phase and the S phase. It is a "point of no return" beyond which the cell is committed to dividing; in yeast this is called START and in multicellular eukaryotes it is termed the restriction point.

2) The G2/M cell stage checkpoint prevents cells from entering mitosis when DNA is damaged; thereby producing an opportunity for DNA repair and
} stopping the proliferation of damaged cells. 


\section{Membrane potential in cancer therapy and emerging microtubule-related treatments.}

Neoplasia is characterized by abnormal proliferation of cells and is closely tied to alterations in membrane potential and unique bioelectrical properties of tumor tissue. Two distinct ionic pathways of action have been documented in currently available literature:

Potassium channels,

Proton, chloride and sodium flux.

Although documented, the resulting physiological role of $V_{\text {men }}$ due to alterations of the above pathways is still not well understood. However, when compared to healthy tissue types, cancer cells exhibit overall depolarization. Thus, hyperpolarization therapy might be a feasible therapeutic method in the future.

Another possible pathway for controlling and/or arresting (cancer) cell proliferation stems from the idea of a microtubule ${ }^{3}$ related mechanism, pictured in Fig.1.

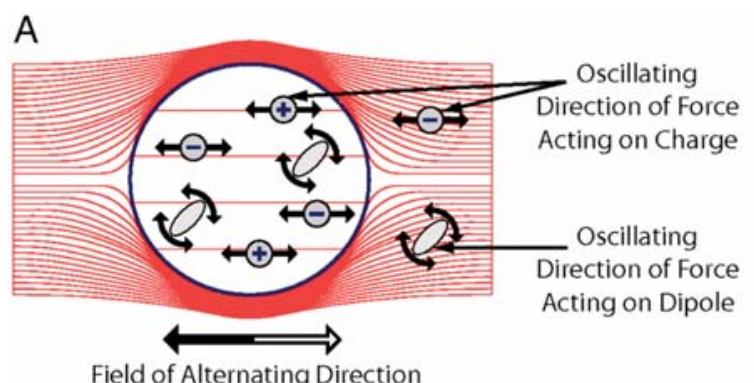

Field of Alternating Direction

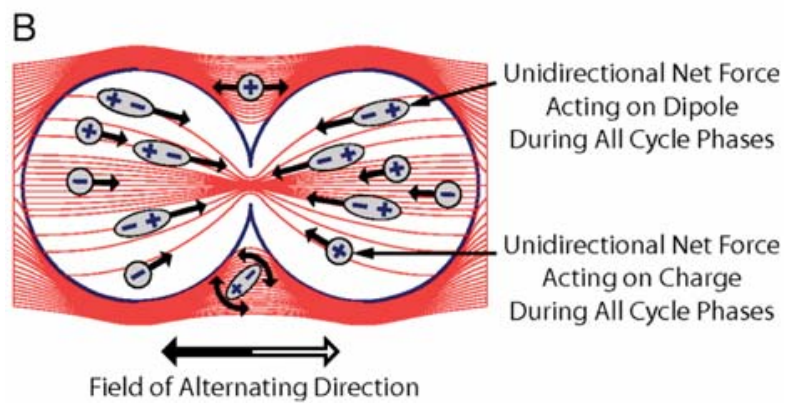

Fig. 1. Idea behind the anti-microtubule mechanism [22]

Two distinct cell phases are shown - quiescent, Fig.1A, wherein the AC field is uniform within the cell and during cellular division, Fig.1B wherein the non-uniform $\mathrm{AC}$ field induces forces that result in the "propulsion" of dipoles towards the furrow. Optima orientation of the applied field (i.e. parallel) is crucial in this case, however also relatively difficult to achieve due to the randomness of the division axes in multiple cells and the relatively long application time of over 16 hours.

The above proposed mechanism of action was evaluated in numerous trials conducted by Kirson et al. [22] by means of external insulated electrodes. The said trials included treatment of various cancer cell lines, including human breast carcinoma and human non-small-cell lung carcinoma amongst others and favorable results prompted a clinical trial on 10 patients with recurrent glioblastoma. Results indicate that the disease progression and overall survival rate more than doubled with no serious, device-related adverse events reported. However, two possible disadvantages were highlighted - undesirable excitation of muscular tissue (i.e. cardiac) and, more seriously, a possible damage of rapidly diving normal cells, such as those of bone marrow, but these have yet to be evidenced in real-life applications.

\section{Conclusion}

The issue of electromagnetic fields of arbitrary frequencies and biological systems is complex and implications are far reaching. To date, numerous studies have reported mixed results in this area, and the influx of new studies only continues to underline this uncertainty.

However, since the times of first macroscopic observations, focus has slowly progressed onto cellular or sub-cellular space, offering a wealth of options to explore and document. One of these options is the modification of the transmembrane voltage via induced currents (eddy currents) in emerging cancer treatments. Physiological parameters, such as membrane voltage and specific ion content, may also be used as in vivo markers to identify special subpopulations of cell types, including various tumor types.

The other possibility consists of manipulating highly polarized structures such as microtubules found within the cell via electric and/or magnetic fields. Positive observations in this area indicate a viable method for controlling undesirable cell proliferation, including numerous tumor types, though the efficacy and means of application has to be further studied and significantly improved to warrant clinical use thereof.

The detailed understanding of the contribution of transmembrane voltage and microtubule manipulation associated with the cell cycle, and especially the integration of this knowledge into biochemical and genetic mechanisms occurring during complex morphogenesis, will reveal fascinating aspects of interdisciplinary biophysics and will offer significant opportunities for the biomedicine of cell engineering, cancer and regenerative medicine.

\footnotetext{
${ }^{3)}$ Microtubules are part of the cytoskeleton and serve as structural components within cells. They are involved in many cellular processes including mitosis, cytokinesis, and vesicular transport. Their structure is predominantly similar to that of proteins and they are highly polarized.
} 
To conclude, the current state of research discussed in this article still does not offer enough evidence to warrant a unanimous answer on whether possible negative effects of electromagnetic fields outweigh the early-reported positive effects of targeted elec- tromagnetic field exposure or vice-versa. However, with every new study published and further elucidation of mechanisms of action, we might find out sooner than later a satisfactory answer to the question posed in the title of this paper.

\section{References}

[1] JAKUSOVA, V.: UV Radiation and Mobile Communication - Physical Properties, Biological Effecte and Health Protection (in Slovak), Vysoka skola zdravotnictva a socialnej prace Sv. Alzbety, Bratislava, ISBN 978-80-89464-00-5, 2009.

[2] KARIPIDIS, K., GILES, G. et al.: Occupational Exposure to Low Frequency Magnetic Fields and the Risk of Low Grade and High Grade Glioma, Cancer Causes Control 18(3): 305-13, 2007.

[3] ERDAL, N., ERDAL, M.E. et al...Lack of Effect of Extremely Low Frequency Electromagnetic Fields on Cyclin-dependent Kinase 4 inhibitor Gene p18(INK4C) in Electric Energy Workers, Arch Med Res 36(2): 120-123.

[4] JOHANSEN, C., OLSEN, J.H.: Mortality from Amyotrophic Lateral Sclerosis, other Chronic Disorders, and Electric Shocks among Utility Workers, Am J. Epidemiol 148(4): 362-368, 1998.

[5] JOHNSEN, C., OLSEN, J.H.: Risk of Cancer among Danish Utility Workers-A Nationwide Cohort Study. Am. J. Epidemiol. 147(6): 548-555.

[6] MILLER, A.B., TO, T. et al.: Leukemia following Occupational Exposure to 60-Hz Electric and Magnetic Fields among Ontario Electric Utility Workers. Am. J. Epidemiol. 144(2): 150-160, 1996.

[7] WILLET, E., ROMAN, E. et al...Occupational Exposure to Electromagnetic Fields and Acute Leukaemia: Analysis of a Casecontrol Study. Occup Environ Med 2003; 60:577-583.

[8] VERKASALO, P. K., PUKKALA, E. et al.: Risk of Cancer in Finnish Children Living Close to Power Lines. BMJ 307(6909): 895-899, 1993.

[9] MCBRIDE, M.L., GALLAGHER, R.P. et al.: Power-frequency Electric and Magnetic Fields and Risk of Childhood Leukemia in Canada. Am J Epidemiol 149(9): 831-842, 1999.

[10] SAVITZ, D.A., WACHTEL, H. et al.: Case-control Study of Childhood Cancer and Exposure to 60-Hz Magnetic Fields. Am J. Epidemiol 128(1): 21-38, 1988.

[11] LINET, M.S., HATCH, E.E. et al.: Residential Exposure to Magnetic Fields and Acute Lymphoblasticleukemia in Children. $N$ Engl J. Med 337(1): 1-7, 1997.

[12] SCHUZ, J., GRIGAT, J.P. et al.: Childhood Acute Leukaemia and Residential 16.7 Hz Magnetic Fields in Germany. Br J. Cancer 84(5): 697-699, 2001.

[13] HENSHAW, D. L., REITER, R.J.: Do Magnetic Fields Cause Increased Risk of Childhood Leukemia via Melatonin Disruption?, Bioelectromagnetics Suppl 7: S86-97, 2005.

[14] VIANALE, G., REALE, M. et al.: Extremely Low Frequency Electromagnetic Field Enhances Human Keratinocyte Cell Growth and Decreases Proinflammatory Chemokine Production, Br J. Dermatol 158(6): 1189-1196, 2008.

[15] LISI, A., FOLETTI, A. et al.: Extremely Low Frequency 7 Hz 100 microT Electromagnetic Radiation Promotes Differentiation in the Human Epithelial Cell Line HaCaT. Electromagn Biol Med 25(4): 269-280, 2006.

[16] CRICENTI, A., GENEROSI, R. et al.: Low-frequency Electromagnetic Field Effects on Functional Groups in Hhuman Skin Keratinocytes Cells Revealed by IR-SNOM. J. Microsc 229 (Pt 3): 551-554, 2008.

[17] SELVAM, R., GANESAN, K. et al.: LF and Low Intensity Pulsed EM Field Exerts its Antiinflammatory Effect through Restoration of Plasma Membrane Calcium ATPase Activity. Life Sci 80(26): 2403-2410, 2007.

[18] VARANI, K., DE MATTEI, M. et al.: Characterization of Adenosine Receptors in Bovine Chondrocytes and Fibroblast-like Synoviocytes Exposed to Low Frequency Low Energy Pulsed Electromagnetic Fields. Osteoarthritis Cartilage 16(3): 292-304, 2008.

[19] ZHANG, X., ZHANG, J. et al.: Effects of Different Extremely Low-frequency Electromagnetic Fields on Osteoblasts. Electromagn Biol Med 26(3): 167-177, 2007.

[20] WEI, Y., XIAOLIN, H. et al.: Effects of Extremely Low-frequency-pulsed Electromagnetic Field on Different-Derived Osteoblastlike Cells. Electromagn Biol Med 27(3): 298-311, 2008.

[21] BLACKISTON, D., MCLAUGHLIN, K. et al.: Bioelectric Controls of Cell Proliferation. Cell cycle 8(21): 3527-3536, 2009.

[22] KIRSON, E., DBALY, V. et al.: Alternating Electric Fields Arrest Cell Proliferation in Animaltumor Models and Human brain tumors." PNAS 104 (24):10152-10157, 2007. 